A 104 MHz RPiFM CAMPUS PUBLIC ADDRESSING BIN

\author{
SHYAMSUNDER MERUGU ${ }^{1}$, RAMCHANDER RAO PAMULAPARTHY ${ }^{2} \&$ J. TARUN KUMAR $^{3}$ \\ ${ }^{I}$ Sumathi Reddy Institute of Technology for Women, Warangal, Telangana, India \\ ${ }^{2,3} S$ R Engineering College, Warangal, Telangana
}

\begin{abstract}
One to one Communication is achieved by mobile networks which are widespread and available across the nation. Yet, they are existing in need to address a specific group is a quantum of people during several occasions which are version controlled and available through a specific region. In this work we have developed a frequency modulated distance controlled public addressing system which can be used as content delivering mechanism or even as a Campus Radio as academic institutions are curious to launch this system at a specific frequency i.e., $104 \mathrm{MHz}$ at SRITW. The campus addressing system is a cost effective and requires low power runs on a Raspberry Pi board along with power supply, SD card, speaker, and antenna. This paper also addresses the future developments for the proposed system.

KEYWORDS: Campus Radio, Raspberry Pi, SD Card, Antenna
\end{abstract}

Received: Jun 06, 2020; Accepted: Jun 26, 2020; Published: Jul 30, 2020; Paper Id.: IJMPERDJUN2020540

\title{
INTRODUCTION
}

The Radio being used for educational purposes since its inception in the early 1920's. One of the applications of radio to the educational problems in developing countries is not a new idea by any means [1]. Many developing countries will be having broadcast capabilities to cover their topographical region. The main technical problem is that the absence of receivers for all individuals. Some countries had legislative problems for use by the populace [2]. Many studies documented the significance of college radio for students in instructional learning, and progress in the campus [3], [4], [5], [6]. There is a requirement for a participatory way to deal with information sharing by the help of new media. [7], [8], [9], [10].

Table 1: Various Applications of Frequency Controlled Public Addressing System

\begin{tabular}{|c|l|l|}
\hline S. No. & \multicolumn{1}{|c|}{ Gratification } & \multicolumn{1}{c|}{ Specific Item } \\
\hline 1 & Education and Learning & $\begin{array}{l}\text { Educational information, tips for exam, assignments, home } \\
\text { works and job interviews }\end{array}$ \\
\hline 2 & Information & $\begin{array}{l}\text { Announcements by Head of the Institute- Team, Information } \\
\text { regarding events in the college. }\end{array}$ \\
\hline 3 & Participation & $\begin{array}{l}\text { Associate with instructors and different understudies (sharing } \\
\text { and learning) } \\
\text { conversations among understudies (sharing and learning) }\end{array}$ \\
\hline 4 & Entertainment & Content produced by team of campus Radio System \\
\hline
\end{tabular}

It was serenely reasoned that college radio is a boundless student development learning medium [11].Also, claimed about its scope and possibility, mainly in the local context, qualify a meticulous examination to file possible fulfillments and challenges that arise throughout its working, and to devise an outline that best owsmoreaids, however nourishing into the key purposes of educational organizations pointing towards the all-round evolution of the scholars [12], [13]. 


\section{EXISTING SYSTEM}

There are many attempts to build the FM radio for sharing the information in the campus. Most of the frequency controlled public addressing system simply called as campus radio are built using basic active and passive components. A simple circuit diagram for campus public addressing system is shown.

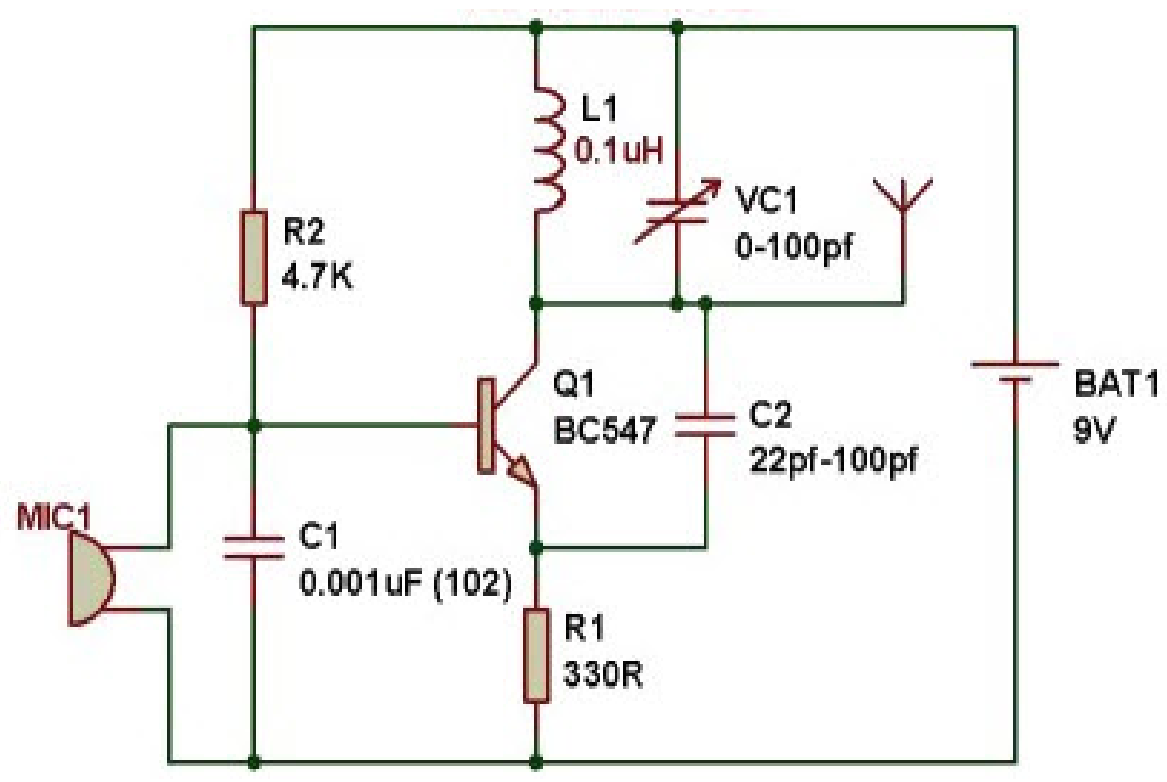

Figure 1: A Simple FM Transmitter.

The fundamental FM transmitter block diagram is shown in figure.1. The above circuit is very simple to design and operate. But at long run, due to the ageing effect of the diverse components used, the working and performance at the desired set frequency is deviated which is not a good concern. So, the things were just moved to develop the same thing using microcontroller devices. The existed transmitter being excellent coverage with less operating power. But, the functioning, and performance of the FM transmitter circuit was highly dependent on the passive components such as induction coil and variable capacitor. The different types of demerits of the existing system include necessity of wider channel, both the transmitter and receiver of FM tend to be more complex due to some interference, consequently there is poor quality in the received signals at the received section. The aging effects of the passive components also play a major role in the working of the campus public addressing system.

The recent FM transmitters were developed by using the Arduino boards where they gave the good results in performance. The FM transmitter uses frequencies of 88-108 MHz for transmitting and receiving the FM signals. The Arduino based FM radio transmitter is shown in figure.2. The entire FM transmitter system is build using a smaller number of components. The various fundamental functional blocks required in an FM transmitter are shown diagrammatically in figure.3. 


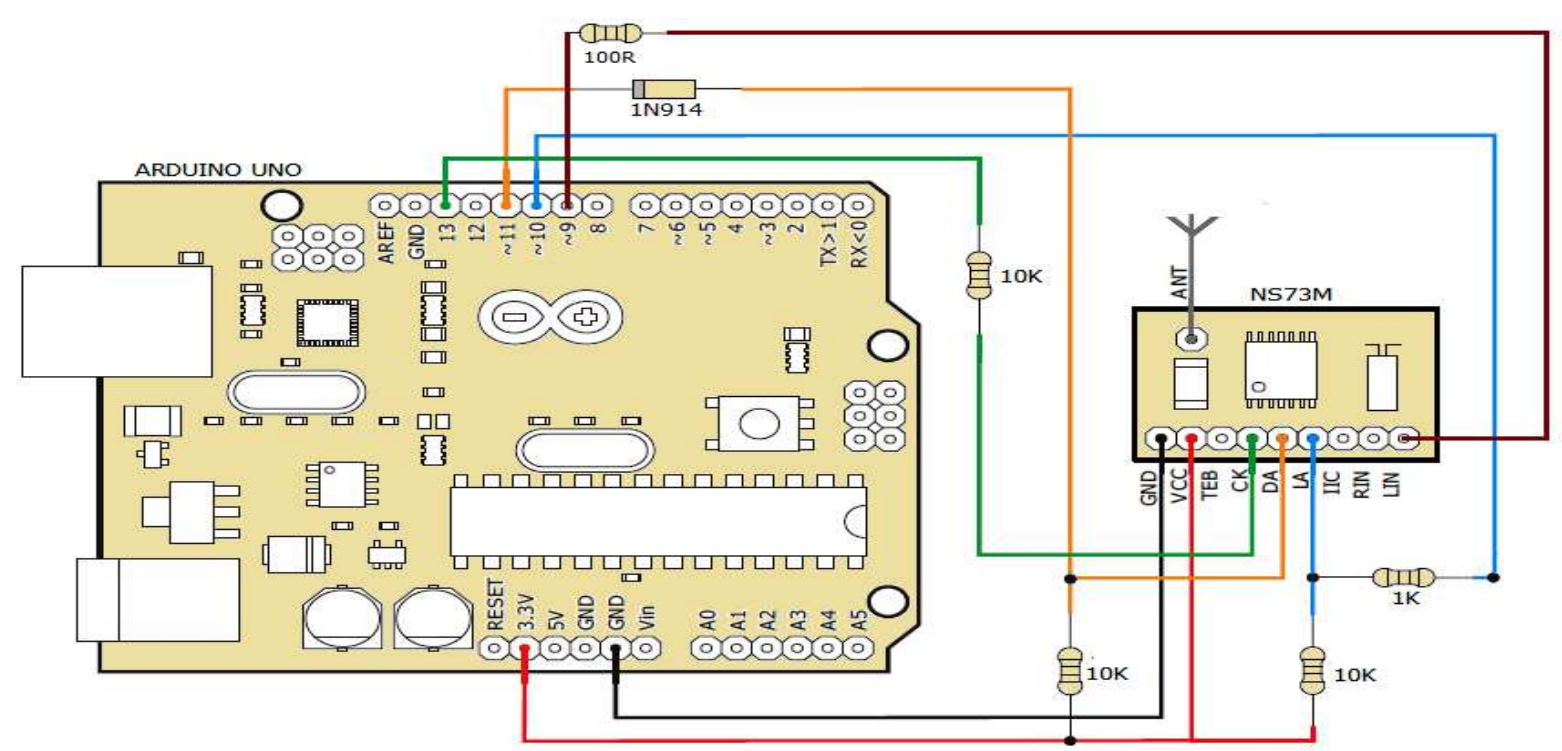

Figure 2: FM Transmitter - Arduino Board Based.

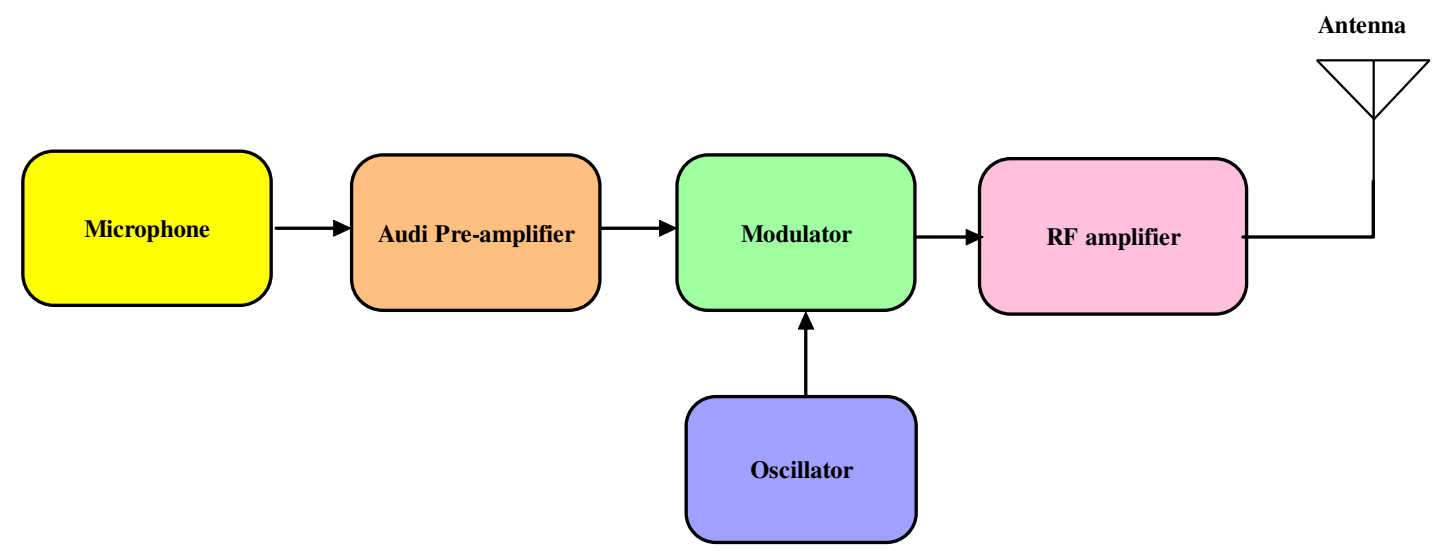

Figure 3: FM Transmitter Block Diagram.

So, here we are proposed an FM transmitter which can overcome the certain disadvantages of the existing system, and is discussed in the subsequent section.

\section{PROPOSED SYSTEM AND COMPONENTS REQUIRED}

The proposed $104 \mathrm{MHz}$, RPiFM Campus Public Addressing System block diagram and required hardware components shown in the corresponding sections below.

\section{Hardware Required}

- $\quad$ Raspberry pi 3b CPU

- $\quad$ Adapter $-5 \mathrm{~V} / 2 \mathrm{~A}$

- $\quad$ SD card 16GB Class 10

- HDMI to VGA cable (VC810 HDMI to VGA converter cable)

- Mobile/ Laptop / Media player 


\section{Software Required}

- $\quad$ Raspbian Operating system

- $\quad$ Python IDE

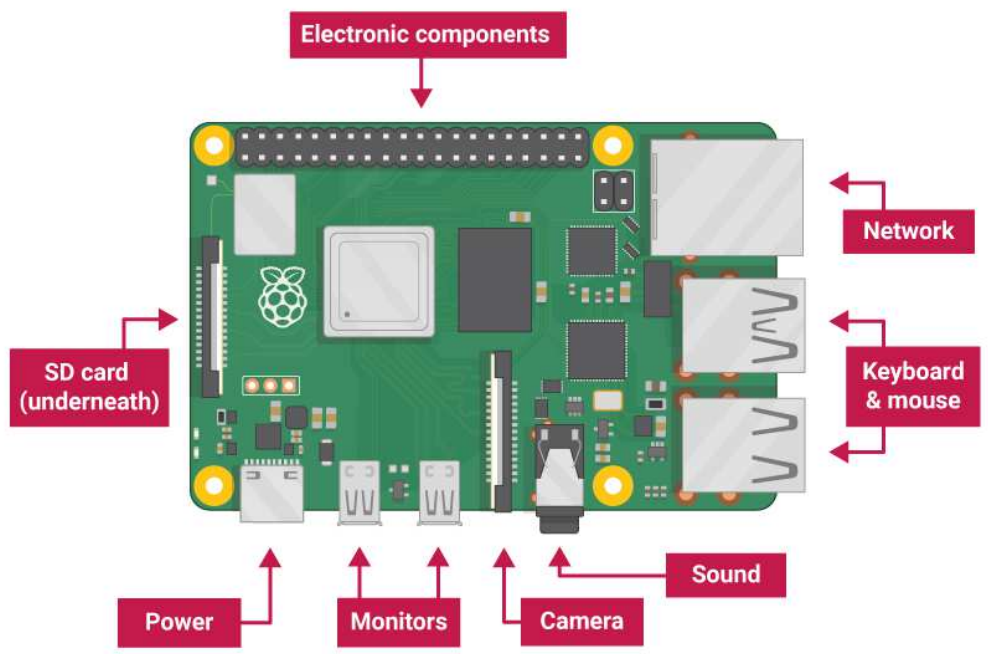

Figure 4: Raspberry Pi Mother Board Connecting Ports Indication Diagram.

The above figure. 3 illustrates the various connecting ports of the Raspberry Pi motherboard. The components have to be connected s per the port indications.

\section{WORKING OF THE CAMPUS RADIO}

This proposed system is implemented as shown in figure. 4. Raspberry pi3 plays a significant role which has total working of CPU it acts as a FM station. The required hardware components have been connected at the respective ports of the Raspberry Pi board. It uses frequency modulation technique in which FM transmitter is employed in this system, where frequency modulation is a process in which the carrier signal frequency is varied with the instantaneous values of the modulating signal. To initiate this system, it needs to set up a channel with certain frequency of 104MHzThen, plugged a strip of wire of few centimeters $(25 \mathrm{~cm})$ into the GPIO4 pin on Raspberry Pi.

For Campus public addressing system, when that particular frequency range is setup, the input that is sent from transmitter has to be in the order, and not coinciding with the existing channels frequency. When it is transmitted it is directly connected Raspberry Pi where the source code written in python helps it in interfacing with the antenna at receiver. Ran the RPiFM code by executing a command like "sudo./pifm rj.wav 104.0", where that "104.0" is the frequency in MHz on which we're broadcasting.

The working of the proposed Campus Public Addressing System is also explained in the form of flow chart. 


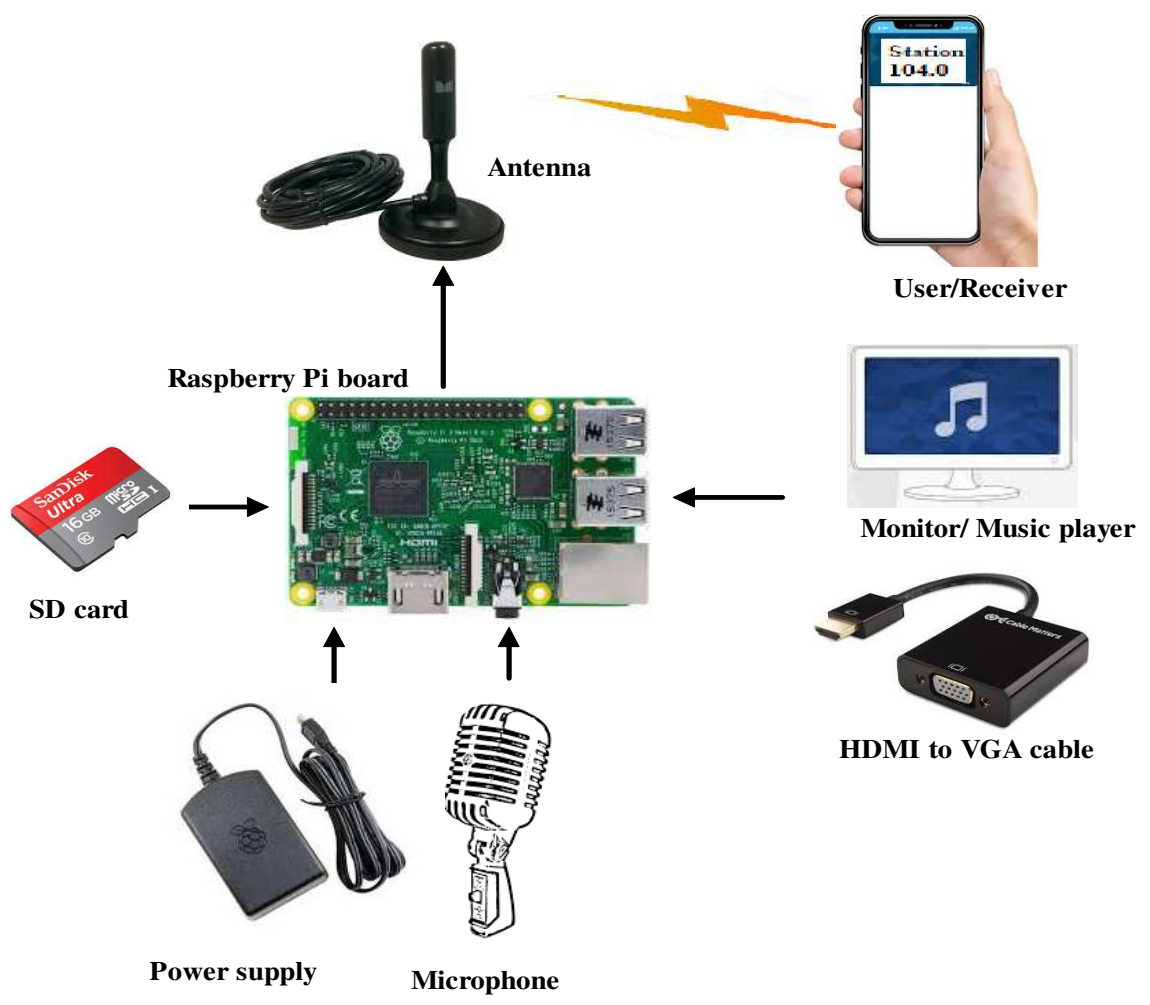

Figure 5: Working Model of the Campus Public Addressing System.

It was so fun to play the pre-recorded audio clips and it was more appealing that live voice is broadcasted using Raspberry Pi. This was simply done by connecting a microphone USB port of Raspberry Pi with a small change in the launch command line of the developed code. The proposed system working is falling into the following fundamental steps.

- $\quad$ Set up Raspberry Pi board

- Install the FM radio software

- Add an antenna

- $\quad$ Choose some music or audio

- $\quad$ Broadcast it

- Turn on the receiver to get the output at the desired frequency of transmission

Here, in this system, the frequency is set to $104 \mathrm{MHz}$ in order to not to collide with the existing systems FM frequencies within the given area of interest. 


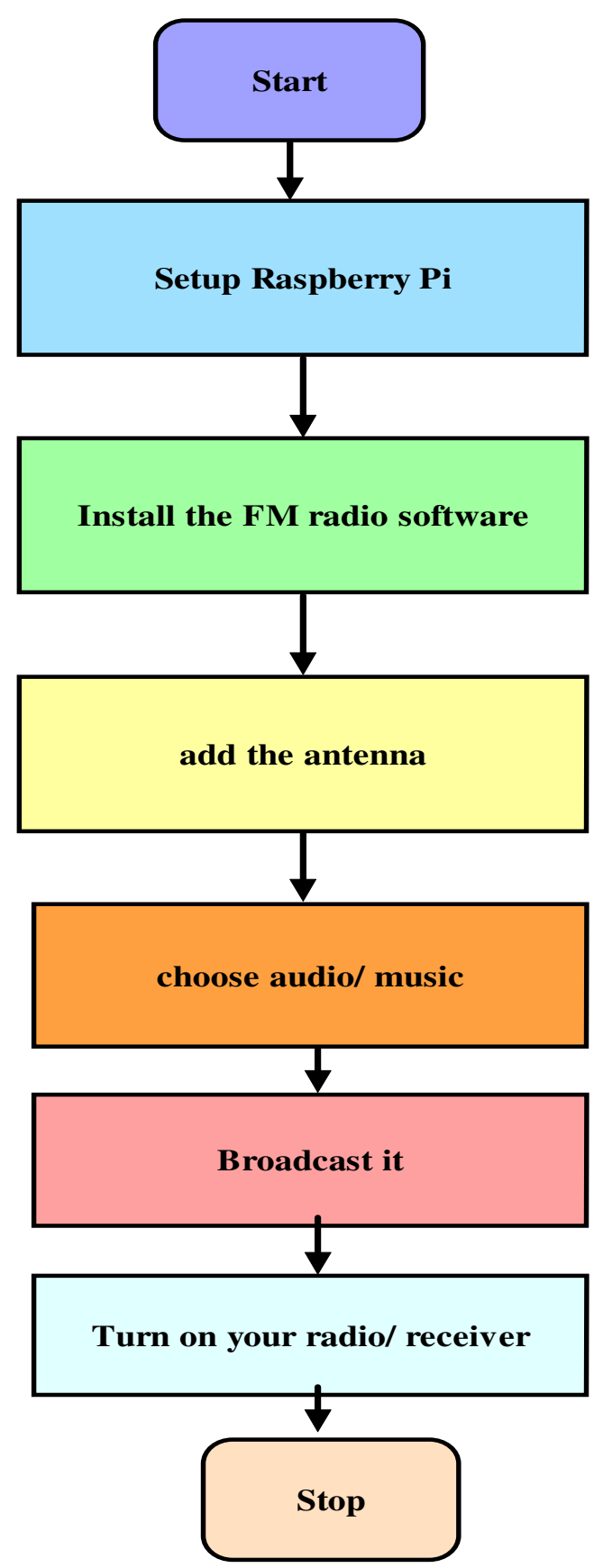

Figure 6: Flowchart at the Transmitter End.

The user at receiver has to tune the frequency that matches with the input frequency $104 \mathrm{MHz}$ that is being transmitted. Here Raspberry Pi serves the whole system. This is implemented in college in order to share the information and for entertainment purpose within the campus.

\section{RESULT}

After setting up the hardware, and running the RPiFM code on Raspberry Pi board, the desired music/ audio file is played at the $104 \mathrm{MHz}$ when the frequency in the mobile/ receiver tuned to that specified frequency, the transmitted signal is received with very minimal noise. 


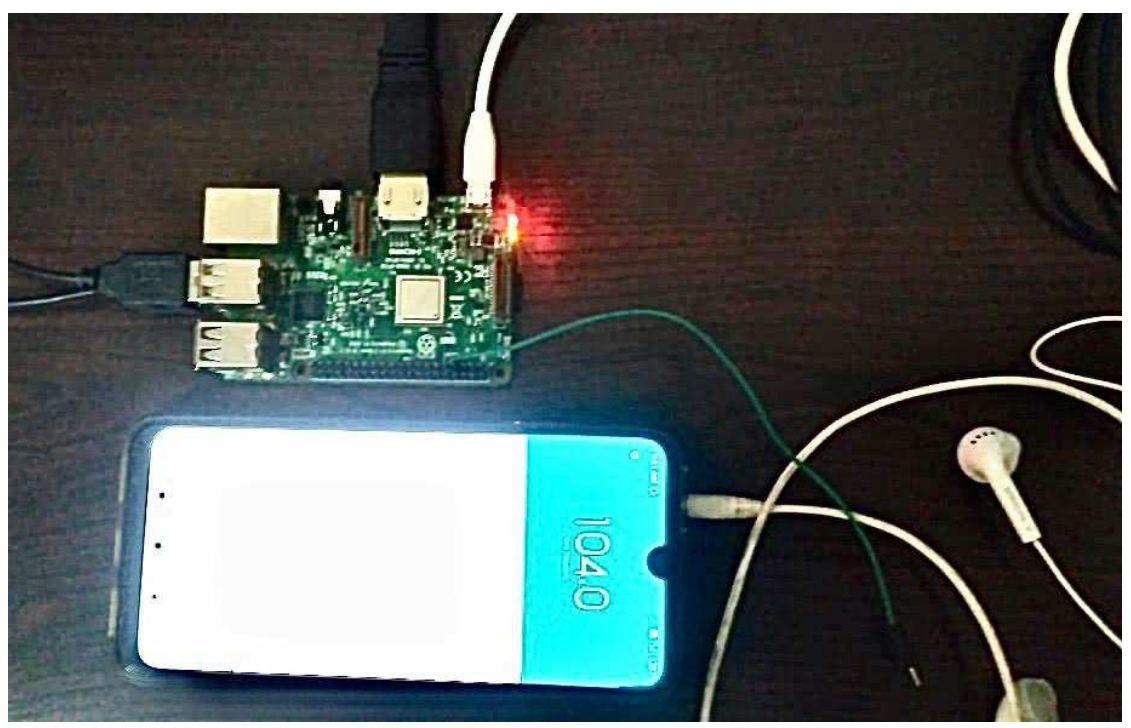

Figure 7: Result of the Proposed System.

\section{CONCLUSIONS}

Here in this paper authors attempted to check the probability of introducing grounds tending to radio framework and its likelihood of gathering among educational institutes in instructive grounds, utilizing constrained yet practical pointers. This paper meant to find the possibilities of school online radio, at Sumathi Reddy Institute of Technology for Women, its need among the understudies, and the conceivable extent of its commitments to understudies' learning, commitment, and data spreading. The investigation is begun from the desire to investigate new measurements to fortify the educational component for an improved learning experience, and furthermore to information sharing among the partners of our organization. It planned for finding the strategy for building up a comprehensive component to anxiety the probabilities of the learning experience by drawing in school open tending to framework as an instructive apparatus. Notwithstanding conventional learning draws near, utilizing RPiFM radio transmitter like an informative medium may restructure the learning environment experiences, and enlarge network commitment. Webcasting can likewise be created in the framework.

Warning: This system was developed for educational and experimental purpose only and not intended to interference any existed FM signals and causing bad effects.

\section{REFERENCES}

1. Ibrahim, B., \& Mishra, N. (2016) - "College radio as a mechanism for participatory learning: Exploring the scope for online radio based learning among undergraduates. Higher Learning Research Communications”, 6(1), 21-34

2. Bueraheng, Nuramal, and Chonlada Laohawiriyanon. "Does learners' degree of exposure to English language influence their collocational knowledge." International journal of english and literature (IJEL) 4.3 (2014): 1-10.

3. Wakefield, M. A., Loken, B., \& Hornik, R. C. (2010) - "Use of mass media campaigns to change health behavior". The Lancet, 376(9748), 1261-1271. 6736(10)60809-4

4. Amber M. K. Smallwood, Bradford L. Yates \& Winston B. Tripp (2018) College Radio Takeovers and Shutdowns: An Analysis of News Coverage from 2006-2016, Journal of Radio \& Audio Media, 25:1, 5-22, DOI: 10.1080/19376529.2017.1361424 
5. Laor, T. (2019). “Journalist 2.0?” educational radio in Israel. Israel Affairs, 25(5), 890-907. doi:10.1080/13537121.2019.1645952

6. Pathak, Bhavna. "The Community Radio Movement in India: Challenges and Opportunities. "International Journal of Communication and Media Studies (IJCMS) 9. 2, Apr 2019, 1-8

7. Hayes, Conor, and Pádraig Cunningham. "Smart Radio - Community Based Music Radio." Knowledge-Based Systems 14, no. 3-4 (June 2001): 197-201. doi:10.1016/s0950-7051(01)00097-1.

8. Laor, Tal, YairGalily, and IlanTamir. “Radio Presence in Online Platforms in Israel.” Israel Affairs 23, no. 5 (July 17, 2017): 951-969. doi:10.1080/13537121.2017.1345420.

9. E. Arunkarthik, and P. Jagadeesh, "PLL based FM transmitter", International Journal of Pure and Applied Mathematics, Volume 119 No. $182018,1429-1434$

10. Moumita Acharya, and Rumpa Biswas(2015) - "Design and Implementation of Frequency Modulated Transmission and Reception of Speech Signal and FPGA Based Enhancement”. ACCENTS Transactions on Image Processing and Computer Vision, Volume-1 Issue-1 November-2015

11. Malur, Prashanth G., and NandiniLakshmikantha."An Exploratory Study on the Opportunities in Visual Media \& the Need to Up Skill Academic Programmes." International Journal for Communication and Media Studies (2019).

12. Prabhakar, V.P. (2012, October)Community Radio Stations - Success stories Retrieved from mediamagazine.in . website: http://mediamagazine.in/content/communityradio-stations---success-stories

13. Dr. OghoghoUyi Osazee-Odia, and Dr. Lucky Ojobor (2017)- “University Students' Listening Behaviour of FM Radio Programmes in Nigeria: An Exploratory Approach". Review of Journalism and Mass Communication, DOI: 10.15640/rjmc.v5n1a3

14. Consultation Paper on Issues related to Community Radio Stations, Telecom Regulatory Authority of India 2014.

15. Chatterjee, Kousik, and Sabuj Dasgupta. "Information Seeking Behavior of Agricultural Researcher While Using Internet: A Case Study of Bidhan Chandra Krishi Viswavidyalaya Central Library, West Bengal, India." West Bengal, India (2016).IASET: International Journal of Library \& Educational Science (IASET: IJLES) 2. 4, Jun - Jul 2016; 11-20

16. Archana R Singh (2010). Community Participation on Campus Radio: A Case Study of Vivek High School 90.4 FM. Retrieved fromhttp://www.caluniv.ac.in/Global\%20mdia\%20journal/Articlejune2010/a\%20r\%20singh\%20-invited.pdf

17. Pinnock, W. J. A. (2014). "Your information station: A Case study of rural radio in the 21st century (Master's thesis)". Department of Communication, Portland State University. 